im Übrigen, ist ein europäischer Grundwert, seit Geltung der Römischen Verträge 1957 europarechtlich verbrieft und seitdem Gegenstand der europäischen Politik, aber insbesondere auch der europäischen Rechtsprechung, der Rechtsprechung durch den Europäischen Gerichtshof, der hier vieles auch ganz konkret in Bewegung gebracht hat; im Vertrag von Rom verankert seit 1957. Es ist aber neben dieser Eigenschaft als Rechtsgrundsatz und Rechtsprinzip, wie bereits angedeutet, auch eine wirtschaftliche Notwendigkeit. Und diese Notwendigkeit gilt nicht nur für die Beschäftigung in der Breite: Sie gilt auch für Spitzenjobs. Ich komme zu einem zweiten Bereich, der neulich in Präsenz von Frau Reding auf diesem Bertelsmann-Symposium angesprochen wurde, nämlich die Vorbildwirkung von Frauen, das Setzen von Vorbildern. Von Leitbildern, auch für junge Frauen, die in Unternehmen arbeiten und die dort durch die Präsenz von Frauen auf dem Top-Niveau, im Top-Management, vor Augen geführt bekommen, dass das möglich ist, dass es erstrebenswert ist. Daneben gibt es natürlich noch viele andere Aspekte, die mit bedacht werden müssen, wie die Vereinbarkeit von Beruf und Familie. Das gilt im Übrigen aber für Frauen genauso wie für Männer. Die Vorbild-Wirkung, die hier durch mehr Frauen in Spitzenpositionen erreicht werden kann, ist eine wichtige Facette, die zu bedenken ist. Das ist vielfach für die nachwachsende Generation die Inspiration; die nachwachsende Generation muss Vorbildern nacheifern können. Frau Reding ist Vizepräsidentin der Kommission - sie hat ein sachliches Portefeuille und dazu gehört die Gleichstellung der Geschlechter - und hat im März dieses Jahres eindringlich an die Chefs der europäischen Konzerne appelliert, ihren Frauenanteil auf den Führungseta- gen zu erhöhen und sich als ersten Schritt zunächst einmal selbst zu verpflichten. Zielvorgabe ist es, bis 2020 einen Anteil von 40 Prozent zu erreichen. Schon im März des kommenden Jahres, so hat sie angekündigt, wird sie prüfen, was bis dahin passiert ist und ob das ausreichend ist. Sie hat außerdem gesetzliche Regelungen angekündigt, sollten die Selbstverpflichtungen nicht zeitnah zum Erfolg führen. Frau Bankier wird das sicherlich im Einzelnen auch noch ausführen. Wir hatten vor wenigen Monaten hier im Hause eine Veranstaltung zu dem Thema mit Vizepräsidentin Reding und mit Frau von der Leyen und beide haben ihre Positionen in diesem Rahmen noch einmal klargemacht.

Heute soll nicht nur die Diskussion allgemein im Mittelpunkt stehen, sondern auch die Präsentation der Studie des Juristinnenbundes. Der Deutsche Juristinnenbund stellt die in dieser Studie zusammengefassten Ergebnisse von 2011 eines sehr interessanten Projektes vor, das deutsche Unternehmen unter die Lupe nimmt und untersucht, inwieweit deren Bemühungen überhaupt erkennbar sind, den Frauenanteil auf der Führungsebene zu erhöhen. Schönen Dank, dass Sie diese Initiative ergriffen haben! Ich glaube, das ist ein sehr wertvoller Beitrag zu einer sachlichen und auch fundierten Diskussion. Wir freuen uns, dieses Projekt hier im Rahmen dieser gemeinsamen Veranstaltung vorstellen zu können und mit Ihnen gemeinsam zu diskutieren. Und damit würde ich mein kleines Grußwort zum Abschluss bringen und an meine Kollegin übergeben. Liebe Frau Bankier, schönen Dank, dass Sie heute aus Brüssel hier eingetroffen sind! Sie werden sicherlich noch sehr viel mehr Substanz beitragen können als ich mit diesen wenigen Worten. Besten Dank!

\title{
Frauenanteil in Führungspositionen: Fakten und Daten aus europäischer Perspektive'
}

\author{
Dr. Daniela Bankier \\ Head of Unit „Gleichstellung von Frauen und Männern“, Gene- \\ raldirektion Justiz, Europäische Kommission, Brüssel
}

Sehr geehrte Mitglieder des Deutschen Bundestages, sehr geehrter Herr Petschke, sehr geehrte Frau Pisal, sehr geehrte Damen und Herren, sehr geehrte Kolleginnen und Kollegen!

Zunächst einmal vielen Dank für den freundlichen Empfang und vielen Dank der Vertretung der Europäischen Kommission und dem Deutschen Juristinnenbund für diese Veranstaltung heute und dafür, dass Sie mir Gelegenheit geben, zum Thema Frauen in Führungspositionen in der Wirtschaft aus europäischer Perspektive zu sprechen. Ich möchte Ihnen in meinem kurzen Impulsreferat einige Fakten und Daten zu diesem Thema aus einer europäischen Perspektive anbieten und möchte damit unsere Diskussion über die wirksamsten Instrumente zur Förderung und zur Sicherstellung einer ausgewoge- nen Präsenz von Männern und Frauen in Führungspositionen einleiten.

Ich beginne mit einer Zahl, die Herr Petschke schon erwähnt hat, und das ist der Anteil von Frauen unter den Hochschulabsolventen in Europa. Dieser liegt heute in Europa bei 60 Prozent. Ich will mit dieser Zahl unterstreichen, dass es sich hier um eine ganz wichtige Talentreserve in Europa handelt, die es zu nutzen gilt. Frauen sind angesichts der demografischen Entwicklung und des Fachkräftemangels in Europa eine wichtige Human Resource. Das klingt ein bisschen profan, weil wir richtigerweise gewohnt sind, Gleichstellung zwischen Männern und Frauen als Grundrecht zu sehen. Aber ich finde es auch wichtig, auf die wirtschaftlichen Nutzen von mehr Gleichstellung hinzuweisen, auf die wirtschaftlichen

\footnotetext{
1 Der Vortrag wurde gehalten im Rahmen der Veranstaltung „Aktionärinnen fordern Gleichberechtigung, Präsentation der Ergebnisse 2011“ am 16.11.2011 im Europäischen Haus, Berlin.
} 
Vorteile, sowohl gesamtwirtschaftlich als auch für die einzelnen Unternehmen. Dieser Nutzen von mehr Gleichstellung ist in mehreren guten Studien bereits untersucht worden, rezent auch in einer hoch interessanten Studie des Bundesministeriums für Familie, Senioren, Frauen und Jugend. Es gibt auch Studien zu diesem Thema von Goldman Sachs, von der Unternehmensberatung McKinsey und von der Deutschen Bank. Es ist sehr wichtig, in die öffentliche Diskussion auch diese wirtschaftlichen Aspekte und die wirtschaftlichen Argumente einzubringen.

Die zweite Zahl, die ich Ihnen nennen will, sind die Beschäftigungsquoten von Männern und Frauen in der Europäischen Union, jeweils mit und ohne Kinder: Die Beschäftigungsquote von Vätern liegt über jener von Männern ohne Kinder und bei den Frauen ist das umgekehrt, da liegt die Beschäftigungsquote von Frauen ohne Kinder über jener von Müttern. In beiden Fällen liegt die Beschäftigungsquote von Männern über der der Frauen. Das deutet darauf hin, dass hier ein großes Potenzial besteht, das wir nicht ausreichend nutzen. Wir müssen die Frauenbeschäftigung sowohl in der Quantität als auch in der Qualität in Europa verbessern.

Ich komme jetzt zum Anteil der Frauen in Führungspositionen in verschiedenen Bereichen des öffentlichen Lebens: Politik, öffentliche Verwaltungen und Wirtschaft. Das Europäische Parlament hat einen Frauenanteil von einem Drittel; die Höchstgerichte in Europa haben einen Frauenanteil von etwas über 30 Prozent; die nationalen, mitgliedstaatlichen Regierungen haben im Durchschnitt einen Anteil von 25 Prozent. Und unter den Vorstandsvorsitzenden von großen, börsennotierten Unternehmen sind nur drei Prozent Frauen. Wir sind also in allen Bereichen sehr weit entfernt von einem Gleichgewicht zwischen Männern und Frauen und die Situation ist besonders krass in den wirtschaftlichen Führungspositionen.

Beim Anteil von Frauen in Aufsichtsräten sieht es so aus: der EU-Durchschnitt liegt bei 12 Prozent. Wir haben also europaweit 12 Prozent Frauen in den Aufsichtsräten der großen, börsennotierten Unternehmen. Es gibt einige Unterschiede zwischen den Mitgliedstaaten, aber keine sehr krassen: Finnland und Schweden bewegen sich bei einem Viertel Frauen in Aufsichtsräten. Auf der anderen Seite der Skala befinden sich Staaten wie Luxemburg, Österreich und Italien, die deutlich unter zehn Prozent liegen. Und Norwegen, ein Land, das nicht zur Europäischen Union gehört, ist fast bei 40 Prozent Frauen in den Aufsichtsräten angekommen. Warum das so ist, erkläre ich gleich. Zuvor noch ganz kurz, wo wir uns im Vergleich zu unseren großen Handelspartnern situieren: Europa hat einen Frauenanteil von 12 Prozent in Aufsichtsräten. Japan, Indien, China, Brasilien sind noch schwächer als Europa und die USA mit 14,5 Prozent ein bisschen, aber nicht sehr viel besser. Also befinden wir uns in guter Gesellschaft.

Jetzt ein paar Informationen zur Tendenz und zur Entwicklung dieser Daten: 2005 kam auf eine Frau in einem Aufsichtsrat neun Männer; 2010 kamen auf eine Frau sieben Männer. Die Tendenz stimmt, es geht Richtung Verbesserung der Situation. Allerdings stimmt das Tempo nicht. Ohne weitere
Maßnahmen, um die Zahl der Frauen in den Vorständen zu erhöhen, wird es weitere 50 Jahre dauern, bis wir zu einem ausgewogenen Verhältnis kommen - und darunter verstehen wir einen Anteil von 40 Prozent Frauen, wir sprechen hier gar nicht von 50 Prozent, sondern von 40 Prozent.

Was tun nun andere europäische Staaten, um diesen Ungleichgewichten etwas entgegenzusetzen? Es gibt verschiedene Lösungsansätze. Es gibt, um das grob zu gliedern, den gesetzlichen Weg, über strikte gesetzliche, verbindliche Quoten, und den Weg der freiwilligen Lösungsansätze. Für die strikten Quoten ist Norwegen sicherlich ein Paradefall. Norwegen hat bereits 2003 ein Gesetz auf den Weg gebracht, das vorsieht, dass 40 Prozent Frauen in den höchsten Entscheidungsgremien der Unternehmen vertreten sein müssen und dieses Gesetz sieht auch Sanktionen vor für den Fall, dass diese Quote nicht erreicht wird. Diese Sanktionen reichen bis hin zur Auflösung des Unternehmens. Die norwegische Quote war ein sehr wirksames Mittel. Es sind heute 40 Prozent Frauen in den Aufsichtsräten in Norwegen vertreten. Das ist über relativ kurze Zeit ermöglicht worden. Ich möchte aber hinzufügen, dass es in Norwegen eine Reihe anderer, begleitender Maßnahmen gegeben hat, um diese Quote zu begleiten und zu unterstützen. Das waren Maßnahmen zur Förderung von Frauen, sowohl auf betrieblicher Ebene, als auch allgemein die Beschäftigung von Frauen.

Dem Beispiel Norwegen sind bislang fünf EU-Mitgliedstaaten gefolgt, die in jüngerer Zeit Quotenregelungen erlassen haben: das sind Frankreich, Belgien, Italien, Spanien und die Niederlande. Alternativ haben eine Reihe von europäischen Staaten den Weg der freiwilligen Lösungen, des Soft Laws, beschritten. Sehr oft sind diese Selbstverpflichtungen in den Corporate Governance Kodices verankert, es handelt sich sehr oft um Empfehlungen zum Frauenanteil; und diese Empfehlungen, das Erreichen dieser Quoten, werden regelmäßig überprüft und oft durch Berichtspflichten nach dem Motto „comply or explain“ begleitet.

Was ist nun der Ansatz der Europäischen Kommission? Wie bereits erwähnt, hat die Europäische Kommission die Erhöhung des Frauenanteils in Entscheidungsgremien als eine von fünf Prioritäten in ihrer neuen Strategie für die Gleichstellung von Männern und Frauen definiert. Kommissionsvizepräsidentin Viviane Reding hat am 1. März 2011 Vorstandsvorsitzende und Aufsichtsratsvorsitzende der großen europäischen Unternehmen nach Brüssel zu einer Diskussion eingeladen, um die Unterrepräsentation von Frauen zu thematisieren und zu diskutieren. Sie hat daraufhin ein Instrument vorgeschlagen, den so genannten „women on the boards pledge“: Das ist eine freiwillige Selbstverpflichtung, die Unternehmen unterzeichnen können und sich damit verpflichten, einen Frauenanteil in Aufsichtsräten und Vorständen auf 30 Prozent bis 2015 und auf 40 Prozent bis 2020 zu erhöhen.

Wie geht es weiter? Die Europäische Kommission wird im März 2012, also sehr bald, Bilanz ziehen. Sie wird dann die Situation und die Fortschritte analysieren und beurteilen und auf der Grundlage dieser Analyse wird sie entscheiden, ob wei- 
tere Maßnahmen notwendig sind auf europäischer Ebene, um die Situation von Frauen in Führungspositionen in Europa zu verbessern. Es gilt also zuerst, Bilanz zu ziehen und dann über weitere Maßnahmen zu entscheiden. Bis dahin setzt die Kommission auch noch auf andere Aktivitäten in diesem Bereich. Wir haben in den letzten Jahren eine Datenbank zu Frauen und Männern in Entscheidungspositionen aufgebaut. Wir sammeln, analysieren und publizieren regelmäßig vergleichbare Daten. Diese Daten sind auch im Internet zugänglich. Wir versuchen auch, Netzwerke zwischen Akteuren zu fördern: Wir haben ein Netzwerk von Frauen in Führungspositionen in der Politik und der Wirtschaft aufgebaut und betreiben das - der Europäische Juristinnenbund (EWLA) ist Mitglied dieses Netzwerkes - und wir versuchen den Austausch guter Praktiken auch zu diesem Thema zwischen den Mitgliedstaaten zu fördern.

Bevor ich schließe, möchte ich dem Deutschen Juristinnenbund meinen Dank aussprechen für die Studie, die heute vorgestellt wird. Solche Initiativen und solche Studien sind sehr wichtig und leisten einen ganz wesentlichen Beitrag zur Gleichstellung von Frauen und Männern im Arbeitsleben. Vielen Dank!

\section{Erhöhung des Frauenanteils in Führungspositionen im europäischen Kontext \\ Vorstellung der Studie 2011 am 16. November 2011 im Europäischen Haus, Berlin}

\section{Ramona Pisal \\ Präsidentin des djb, Berlin}

Auch ich begrüße Sie im Namen des Deutschen Juristinnenbundes sehr herzlich, sehr geehrter Herr Petschke, sehr geehrte Frau Bankier, sehr geehrte Damen und Herren, liebe Kolleginnen im Deutschen Juristinnenbund, liebe Gäste, liebe Anwesende!

Haben Sie vielen Dank für die freundliche Begrüßung hier in Ihrem Haus Herr Petschke. Als dem Hausherrn und Leiter der Vertretung der Europäischen Kommission in Berlin sind wir Ihnen besonders dankbar, dass Sie uns hier bereitwillig Gastfreundschaft gewähren. Auch vielen Dank für Ihre einführenden Worte in das Thema. Vielen Dank, Frau Bankier, für das Statement, das uns auch schon in den europäischen Kontext gestellt hat, und auch an Sie, dass Sie mit Ihrer Gegenwart hier und der Einladung, die in dieses Haus erfolgt ist, uns den Rahmen geben und das Gewicht, das - wie wir meinen und wie viele meinen - dieser Aktion und der Idee, die damit verbunden ist, auch zusteht. (...)

Meine Aufgabe ist es heute, Ihnen unsere Studie vorzustellen, die auch ich eben, wie Sie sie jetzt auf den Stühlen liegen haben, zum ersten Mal in gedruckter Form sehe. Sie ist wunderbar gelungen, äußerlich und auch inhaltlich sehr gewichtig. Für Sie ist es kein Geheimnis und Herr Petschke hat es eben herausgestellt: Der Deutsche Juristinnenbund setzt sich nicht erst neuerdings, sondern schon seit Jahrzehnten dafür ein, dass Frauen in Führungspositionen auch in der Wirtschaft maßgeblich vertreten sind. Es hat allerdings auch Jahrzehnte lang - und insbesondere sprechen wir da vom letzten Jahrzehnt - trotz Appellen auf nationaler und europäischer Ebene keine signifikante Besserung, was diese Repräsentanz anlangt, ergeben. Nach wie vor lässt sich also uni sono konstatieren, dass der Stand der Frauen in Führungspositionen in der Wirtschaft auf einem inakzeptabel niedrigen Niveau stagniert.
Das wollen wir mit diesem Projekt verändern, indem wir Fragen stellen. Wir haben dieses Projekt 2009 begonnen. Die erste Studie haben wir nach der ersten Besuchsrunde von 75 Dax-Unternehmen im Dezember 2010 vorgestellt: Sie zeigt, dass die Unternehmen bislang keine geeigneten Maßnahmen ergriffen haben, die Repräsentanz von Frauen zu erhöhen. Heute hier mit der Studie des zweiten Durchgangs freue ich mich, Ihnen auch positive Tendenzen vorstellen zu können.

Wie Deutschland im Vergleich dasteht, hat Ihnen eben Frau Bankier schon berichtet. Ich füge noch zwei Zahlen hinzu: Innerhalb der 27 EU-Staaten sind wir auf dem neunten Platz bei den Aufsichtsräten und dieser im Vergleich gute Platz ist geschuldet der Tatsache, dass von Arbeitnehmerseite oft Frauen in die Aufsichtsräte entsandt werden. Bei den Vorständen sieht es jedoch sehr, sehr schlecht aus: Deutschland liegt mit zwei Prozent im internationalen Vergleich auf einem der letzten Plätze.

Unsere Studie haben wir in diesem Jahr in einen europäischen Kontext gestellt. Sie heißt „Aktionärinnen fordern Gleichberechtigung 2011 - Erhöhung des Frauenanteils in Führungspositionen im europäischen Kontext" und geht in vielen Einzelbeiträgen auf das Thema Europa ein. Kernstück ist die Auswertung der von uns besuchten Hauptversammlungen. Diese wird ergänzt durch Beiträge, die sich mit der Situation in Deutschland, in Europa und in einzelnen europäischen Ländern befassen, und schließlich legen einige Unternehmen ihre Personalstrategien dar.

Der Stand der Aktivitäten auf europäischer Ebene nimmt in der Studie 2011 also einen breiten Raum ein - das politische Programm der Barroso II-Kommission, die Initiative der Vizepräsidentin Reding. Wir wissen alle, dass Norwegen das Vorreiter-Land ist, weil es 2003 ein entsprechendes Gesetz erlassen hat. Wir nehmen in unserer Studie sachverständig zu elf Ländern mit 14 Beiträgen Stellung. Drei dieser Beiträge befassen sich mit Norwegen, denn das Land bietet mit seiner 